\title{
JORGE CRISTIAN SCHYTHE: EL GOBERNADOR (1853-1858) Y METEORÓLOGO DE PUNTA ARENAS, REGIÓN DE MAGALLANES ${ }^{1}$
}

\author{
JORGE CRISTIAN SCHYTHE: THE GOVERNOR (1853-1858) AND \\ METEOROLOGIST AT PUNTA ARENAS, MAGELLAN REGION
}

Vicente Pérez ${ }^{2}$

Magallanes se constituye en "Territorio de Colonización" el 8 de julio de 1853, por Decreto del Presidente don Manuel Montt y su Ministro Antonio Varas y el 15 de agosto del mismo año, don Jorge Cristian Schythe, danés, profesor de Ciencias Naturales, asume la gobernación magallánica con residencia en Punta Arenas (Martinic 1992). En 1853 la colonia tenía sólo 151 habitantes, incluido en este número 71 niños (Irarrázaval 1966); en 1854, serían 153 habitantes (Martinic 1992).

Varias grafías diferentes se asignaron al apellido del Gobernador: Schytte (Domeyko 1854, del Barrio 1856), Schyte (Domeyko 1859), Scythe (Drault 2012); también Punta Arenas: Punta-Arenas (Domeyko 1859, 1872b).

Del Barrio (1856), en la misma línea de pensamiento que Schythe, propugna que el estudio de la meteorología sería de gran beneficio para mejorar la agricultura, el desarrollo de la higiene pública y proporcionar bases para abrir las puertas a la inmigración y la colonización. Y enfatiza en la necesidad de uniformarlas.
Domeyko (1872a, 1872b) destaca el hecho de que el nuevo gobernador, a pesar del tiempo que debe dedicar a sus labores administrativas, ha logrado llevar un registro minucioso de observaciones barométricas y termométricas del estado atmosférico, de las lluvias y direcciones de los vientos; y que importa conocer estas características de esta extremidad meridional del territorio chileno por su importancia "no solamente para el estudio de la meteorología de Chile, sino también para el de la física general de nuestro planeta".

En esta nota, se proporcionan los fundamentos para apreciar la importancia de las observaciones de Jorge Cristian Schythe para el desarrollo de la meteorología del país, valorar la rigurosidad de su trabajo realizado que tenía una finalidad aplicada, comprender el porqué de la acogida que le brindó la Universidad de Chile y, finalmente, ordenar la catalogación de sus publicaciones.

Para ello, se revisaron, en primer lugar, los aportes bibliográficos de Anales de la Universidad de Chile (1859), Anónimo (1995), Rebolledo

1 Perteneciente al proyecto "Antecedentes para la Historia de las Ciencias Naturales en la Región de Magallanes".

2 Laboratorio de Entomología, Instituto de la Patagonia, Universidad de Magallanes, Punta Arenas. vicente.perez@umag.cl. 
(1995) y Sanhueza (1995), que contribuyen a explicar y resolver problemas estructurales de edición de los Anales mencionados y corrigen errores de indexación. Asimismo, se ubicaron los trabajos publicados por Schythe (1854, 1855, 1856a, 1856b, 1856 c, 1862, 1863a, 1863b) y Burns (1859) en la mencionada serie periódica de la Universidad de Chile.

No fue posible ubicar las cartas intercambiadas entre Schythe y Domeyko y de cuya existencia da cuenta éste último (Domeyko 1859).

Los principales resultados y conclusiones a las cuales es posible llegar luego de la exhaustiva revisión bibliográfica, son los siguientes:

- Schythe registra publicaciones entre 1854 y 1863, todas ellas en los Anales de la Universidad de Chile. Durante una ausencia de Punta Arenas, las observaciones meteorológicas que había iniciado fueron efectuadas por el cirujano de la Armada J.R. Burns (1859).

- Domeyko (1872b) recuerda que las primeras observaciones meteorológicas en Punta Arenas abarcaron sólo cinco meses, desde el 1 de junio de 1851 hasta el 27 de octubre del mismo año y se debieron al Gobernador Benjamín Muñoz Gamero (Muñoz Gamero 1852), muerto en 1851. Comenta sí, que, desgraciadamente, el aneroide utilizado por Muñoz Gamero no estaba, aparentemente, bien calibrado y carecía de la sensibilidad para detectar las variaciones diurnas.

- Schythe era muy estricto en lo referente a observaciones meteorológicas. Su expresión críptica en nota a pie de página (Schythe 1856) fue una clara alusión a su predecesor en la Gobernación:"Las observaciones meteorolójicas hechas en Punta Arenas desde el 1 de junio hasta el 27 de octubre de 1851, publicadas en los "Anales de la Universidad de Chile" el 30 de junio de 1852 , soi de opinión que no merecen ningún crédito, reservándome para otra ocasión el esponer las razones que tengo para considerarlas en parte fingidas y del todo inexactas" (la cursiva es nuestra). Nunca expuso sus razones.

- El Decano de la Facultad de Ciencias Físicas y Matemáticas de la Universidad de Chile (1856), en Sesión del 16 de agosto da cuenta que la Facultad acordó proponer al Supremo Gobierno que se designe al Gobernador de la Colonia de Magallanes, don Jorge Schythe, como Miembro Co- rrespondiente, por haberse distinguido publicando trabajos científicos sobre Magallanes.

- En Sesión del 30 de agosto del mismo año se dio cuenta de un Oficio del Ministro de Instrucción Pública en que se transcribe el Decreto Supremo en el que se incluye como Miembro Correspondiente de la Facultad a don Jorge Schythe.

- Domeyko (1859) destaca que el señor Schythe es "un observador muy prolijo", al que se debe "una serie muy interesante de observaciones meteorológicas en Punta Arenas".

- Geise (1861), al comparar características climáticas de varias regiones del país, alude a "los datos exactos que poseemos del Estrecho de Magallanes".

- Schythe (1854) en un párrafo titulado "Advertencia" explica la metodología que emplea ("los barómetros i termómetros están colgados a la sombra hacia el Sur, en la pared de un cuarto en que no se hace fuego"), describe los instrumentos que utiliza: barómetros (francés, inglés, aneroide), termómetros (centígrado y Farenheit), pluviómetro y veleta; expresa (Schythe 1856) sus registros en Tablas que incluyen día del mes, hora, barómetro, viento, tiempo, aguas y notas. Es decir, incluye lo que hoy expresamos como materiales, métodos y resultados en un artículo científico.

- Las observaciones meteorológicas de la región de Magallanes llamaban la atención en la Universidad de Chile, ya que eran muy diferentes a las del resto del país. Así, Domeyko (1859) manifiesta su asombro de que Schythe "no ha sentido un solo temblor" y "no ha visto ni una sola vez indicio de aurora austral". Esto mismo lo repite posteriormente (Domeyko 1872). Hay que tener presente que entre las fantasías sobre el extremo meridional de Chile estaba la de "los volcanes rugientes de Tierra del Fuego" (Malte-Brun 1853).

- Las observaciones meteorológicas de Schythe son las únicas comentadas. Así, en Schythe (1854), al final hay una Nota relativa a las observaciones mandadas por el Sr. Schythe, de la cual puede ser autor Domeyko: “ 1) Las observaciones barométricas, la altura barométrica media del lugar en el nivel del mar, es notablemente inferior a lo que se ha notado hasta ahora en otras partes del mundo; 2) Los miembros más frecuentes en el Estrecho de Magallanes son occidentales; 3) La temperatura media en los 6 meses ha sido $9,4^{\circ}$..."). 
- Domeyko expresó repetidamente su admiración por el trabajo de Schythe, tanto que reprodujo textualmente el mismo párrafo referente a él en dos oportunidades (Domeyko 1872b, 1875). Este último, por error fue numerado volumen 47 de los Anales (Sanhueza 1995).

- El hecho de haber podido publicar en los Anales de la Universidad de Chile proyectó a Schythe en el ámbito internacional, y así fue incluido en la lista de los grandes compiladores bibliográficos como Brockhaus (1858), que registró a Schythe (1856a), aunque erróneamente indicó "hasta junio de 1856".

- No se puede consultar la última publicación: Schythe (1863b): al final de la página 518 del volumen 23, la Redacción advierte: "Insertamos a continuación varias Observaciones Meteorológicas", indicadas con numerales de $1^{\circ}$ a $7^{\circ}$, de las cuales la sexta corresponde a Punta Arenas, de Schythe, página 533 (inicio) sin límites de inicio unas y de término otras (son solamente Tablas).

- El homenaje del Instituto de la Patagonia de la Universidad de Magallanes en Punta Arenas está en la existencia de la Estación Jorge Schythe (5308' S; 7053' W; 6 m.s.n.m.), con cuyos datos se publica anualmente un Resumen Meteorológico en los Anales del Instituto de la Patagonia.

\section{LITERATURA CITADA}

Anónimo. 1995. Los "Anales de la Universidad de Chile" en la Historia. Anales de la Universidad de Chile, Sexta Serie, 1:12-15. h ttp:// www.revistas, uchile.cl/index.php/ANU/issue/view/351. 10/03/2012

Anales de la Universidad de Chile. 1859. Instrucción para la acertada encuadernación de la colección completa de este período. Anales de la Universidad de Chile, 16:610-614

Brockhaus, F.A. 1858. Allgemeine Bibliographie 8. Berlin.

Burns, J.R. 1859. Observaciones meteorológicas tomadas en la Colonia de Punta-Arenas (Estrecho de Magallanes) por el término de un año, contado desde el lunes 8 de junio de 1857 hasta el 8 de junio de 1858, inclusiva. Anales de la Universidad de Chile, 16:574579
Decano de la Facultad de Ciencias Físicas y Matemáticas. 1856. Sesión 16 de agosto y Sesión del 30 de agosto. Anales de la Universidad de Chile, 16:202-296.

Del Barrio, P. 1856. Sobre la utilidad que podrían tener en Chile las observaciones meteorológicas; sobre el modo de arreglarlas. Anales de la Universidad de Chile, 13:359-367

Domeyko, I. 1872a. Meteorolojía de Chile. In Memoria presentada al Consejo de la Universidad en sesión de 4 de octubre de 1872 por el señor Rector. Anales de la Universidad de Chile, 41:643-647

Domeyko, I. 1872b. Resumen de las observaciones meteorológicas hechas en diversos lugares del país, desde Atacama hasta el Estrecho de Magallanes. Anales de la Universidad de Chile, 42:635-694

Domeyko, I. 1875. Reseña de los trabajos de la Universidad desde 1855 hasta el presente. Anales de la Universidad de Chile, 48:170-276 http://www.revistas.uchile.cl/ index.php/ANUC/article/viewPDFInterstitial/12011/12370 12/04/2012.

Drault, R. 2012. Patagonia Database - Historia Personas A-Z- Apellidos SAN - SCH. Windows Internet Explorer. Online: http://drault. com/pdb/Personas.A-Z.html 26/03/2012.

Geisse, F. 1861. Ensayo sobre el clima del Territorio de Llanquihue. Anales de la Universidad de Chile, 19:743-757

Irarrázabal, J.M. 1966. Historia de las Relaciones Internacionales de Chile. La Patagonia. Editorial Andrés Bello.

Malte-Brun, C. 1853. Descripción particular de Chile, de Araucanía, de Patagonia y de las Tierras Magallánicas. In La Geografía Universal, o descripción de todas las partes del Mundo. Madrid. 840 pp. http://books.google.co.ve/ books?id=BDBYjviguk8C\&printsec-front cover $\& \mathrm{hl}=$ es\&source=gbs-gi 16/01/2012

Martinic, M. 1992. Historia de la Región Magallánica. Volumen I. Universidad de Magallanes. $781 \mathrm{pp}$.

Muñoz Gamero, B. 1852. Sobre el clima del Estrecho de Magallanes. Observaciones meteorológicas hechas desde el $1^{\circ}$ de junio hasta el 27 de octubre de 1851. Anales de la Universidad de Chile, 9:289-296 
Rebolledo, A. 1995. Consideraciones en torno a los "Anales de la Universidad de Chile". 1842-1879. Anales de la Universidad de Chile, Sexta Serie, 1:23-32 http://www.tecnovet.uchile.cl/index.php/ANUC/article/ viewFile/8265/8018 10/04/2012

Sanhueza, J. 1995. Los "Anales de la Universidad de Chile", su numeración y sus series. Anales de la Universidad de Chile, Sexta Serie, 1:17-21. h ttp://www.revistas.uchile. $\mathrm{cl} /$ index.php/ANUC/article/view/PDFIntersticial/8264/8017 19/04/2012

Schythe, J.C. 1854. Observaciones meteorológicas hechas en Punta Arenas (Estrecho de Magallanes): desde el $1^{\circ}$ de setiembre $\mathrm{d}$ 1854. Anales de la Universidad de Chile, 11:265-301

Schythe, J.C. 1855. El Territorio de Magallanes i su colonización. Anales de la Universidad de Chile, 11:435-465

Schythe, J.C. 1856a. Observaciones hechas en Punta Arenas, Estrecho de Magallanes, desde marzo $1^{\circ}$ de 1855 , hasta agosto último del mismo año. Anales de la Universidad de Chile, 13:115-132. (¿o 137-156?)

Schythe, J.C. 1856b. Observaciones hechas en Punta Arenas, Estrecho de Magallanes, des- de septiembre $1^{\circ}$ de 1855 , hasta febrero último de 1856; acompañadas de un apéndice que comprende las observaciones correspondientes a los meses de marzo, abril, mayo i junio. Anales de la Universidad de Chile, 13:208-226

Schythe, J.C. 1856c. Apéndice a las Observaciones hechas en Punta Arenas, Estrecho de Magallanes, correspondiente a los meses de marzo, abril, mayo i junio de 1856. Anales de la Universidad de Chile, 13:268-279

Schythe, J.C. 1862. Observaciones meteorológicas hechas en Punta Arenas, del territorio de Magallanes, desde octubre $1^{\circ}$ de 1860 hasta marzo último 1861. Anales de la Universidad de Chile, 21:193-209

Schythe, J.C. 1863a. Meteorolojía: Observaciones meteorológicas tomadas en Punta Arenas del territorio de Magallanes, desde el $1^{\circ}$ de octubre de 1861, hasta marzo de 1862. Anales de la Universidad de Chile, 22:226242

Schythe, J.C, 1863b. Meteorolojía. Observaciones meteorológicas tomadas en Punta Arenas desde el $1^{\circ}$ de octubre de 1862 hasta marzo último de 1863. Anales de la Universidad de Chile, 23:533-556 